\title{
Artificial Intelligence in the Field of Oral Medicine - A Systematic Review
}

\author{
Kumar Sougata ${ }^{1}$, Ayushi Bhargava ${ }^{1}$ and Gaurav ${ }^{2 *}$ \\ ${ }^{1}$ House Surgeons, NSVK Sri Venkateshwara Dental College and Hospital, Bangalore, \\ Karnataka, India \\ ${ }^{2}$ Consultant Oral Physician and Maxillofacial Radiologist, Assistant Professor, \\ Department of Oral Medicine and Maxillofacial Radiology, NSVK Sri \\ Venkateshwara Dental College and Hospital, Bangalore, Karnataka, India \\ *Corresponding Author: Gaurav, Consultant Oral Physician and Maxillofacial \\ Radiologist, Assistant Professor, Department of Oral Medicine and Maxillofacial \\ Radiology, NSVK Sri Venkateshwara Dental College and Hospital, Bangalore, \\ Karnataka, India.
}

Received: February 23, 2021

Published: March 20, 2021

(C) All rights are reserved by Gaurav., et al.

\begin{abstract}
Background: With the entire world weaving the cocoon of Robotics and Artificial Intelligence (AI) it's hard to exempt the field of dentistry from the effects of this powerful tool. From venturing into cases involving vital structures to now being associated with the effects of ever going technology dentistry has evolved leaps and bounds. From minimizing harm to the patient to predicting the dental schedule of a teenager, algorithms and softwares are built to do it all.

Aim of The Study: To assess the significance of Artificial Intelligence in the field of Oral Medicine.

Research Question: Is Artificial Intelligence significant in different pathological arenas of Oral Medicine.

Materials and Methods: With the Medline database taken as a source for authenticated scientific research data, articles were selected having undergone Randomized Control Trial. Out of these, articles (studies) were chosen which met the criterion for Systematic Review.

Results and Conclusion: From our study we can interpret that the field of Artificial Intelligence has a huge potential to change the way we look at conventional Oral Medicine functioning today. It can not only serve as an efficient tool to a clinician but also diminish the gap between a healthcare provider and a patient. Most importantly it reduces the burden on our existing healthcare and diagnostic fields. However, there are a number of misapprehensions regarding this emerging technology that needs to addressed and studied. On a whole this field presents with a perfect amalgamation of human mind with computer technology to form a strong backbone for public health and thereby engaging individuals to take care of their own health.
\end{abstract}

Keywords: Artificial Intelligence; Oral Medicine; Robotics

\section{Introduction}

It has taken seven million years of human evolution and countless adaptive skills which have helped the human race to be at the top of animal hierarchy. "Artificial Intelligence" or usage of ma- chines to mimic human cognitive skills and mind has been lingering scientific minds ever since Plato came up with his studies of "Neural Networks" and "Theory of Forms" [1-3] in 400 B.C. Ever since then scientists and philosophers have envisaged the idea of 
using artificial neural networks to mimic human-like behavior and skills. However, it wasn't until 1956's Dartmouth conference that the word "Artificial Intelligence", coined by John McCarthy [4,5] gained significant popularity in the research community. This conference succeeded the famed "Turing test" or the "Imitation Game" [6] performed in the year 1950 by British mathematician Alan Turing which puts forth the idea of testing a machine's ability and to prove whether or not a given machine is either similar or presents itself as an indistinguishable from of a human mind.

\section{Aim of the Study}

To assess the significance of Artificial Intelligence in the field of Oral Medicine.

\section{Research Question}

Is Artificial Intelligence significant in different arenas of Oral Medicine?

\section{Materials and Methods}

Various researches and studies have documented Artificial Intelligence to be sensitive and specific. With this fact in mind, a literature-based systematic review was carried out to fulfill the aim of the study. With Cochrane collaboration taken as a source for authenticated scientific research data, about 30 research articles were selected; having undergone randomized control trial. Out of these, the articles were screened and finally, 25 articles were chosen which met the criterion for a systematic review.

\section{Result}

The following conclusions were drawn from the final 25 articles selected and the foundation of this systematic review was based on these conclusions.

In the next few decades, Artificial Intelligence would be tightly woven into the fabric of healthcare data and their processing, as large volumes of information can be extracted from these data records to make real-time inferences for health risks and health outcome predictions.

These systems can reduce the risks of therapeutic errors which are inevitable in human daily practices while preventing conditions such as "Observational Fatigue" at minute single-pixel levels.

Incorporation of Artificial intelligence in teaching and learning processes can dramatically improvise the way students especially in medical and dental backgrounds perceive knowledge.
Nevertheless, this technology is helping create more awareness pertaining not only to oral and maxillofacial diseases but also in various other medical conditions which otherwise would have been classified under dubious diagnosis.

The incorporation of Artificial intelligence in the field of oral and general pathology has allowed accurate detection of occult metastasis and comparative analysis of immunohistochemistry and other techniques eliminating subjective and observational bias.

While we navigate these complicated times of the COVID pandemic Artificial Intelligence is constantly helping us in all ways from data processing to maintaining patient records from vaccine research developments to assisting clinicians with diagnostic decision making.

\section{Discussion \\ Background}

The intersection of Artificial intelligence and medicine first occurred in the year 1964 when "Eliza" [7] was introduced; it became the first chatbot or a conversational tool to recreate the conversations between a psychotherapist and patient. It was then followed by the introduction of "Dendral" [8], created by a group of scientists at Stanford; Dendral is used to date by pharmaceutical companies to store details of their organic compounds. It was not long since then when CAD/CAM [around the year 1970] was introduced in dentistry which revolutionized dental treatments.

HERMES [9] Operating room or the Control Centre was introduced by Computer Motion Inc. and cleared by the FDA on (April 1998) which allowed networking the medical equipment kept in the operating room and allowing surgeons to have direct control over these devices using verbal commands. AESOP was introduced and cleared by the FDA in the year 1994 which assisted the surgeons in minimally invasive procedures. It was around this time that the FDA also cleared the Zeus Robotic Surgical System [9]. In the year 2003 Computer Motion Inc. merged with Intuitive Surgical to introduce the Da Vinci Surgical System [10] which proved to be a landmark in the field of surgical science. DEXvoice was introduced in the year 2015 by Simplify eye [11] (a virtual add-on module to the DEXIS imaging software that allows customers to use any Amazon Alexa enabled platform to integrate voice activation to their dental workflow. Imagine a dentist using their voice in the operatory to capture, show, compare, take, and/or acquire images). Through acquiring the idea of machine learning and 
processing, MMG Fusion introduced ChairFill, an algorithm [11] that retrieves data from dentists and analyzes them, if trained in the future it can initiate a direct patient conversation pattern and even book appointments without any human involvement. Another company using a similar algorithm is Dentistry.ai; it is designed to take a large set of radiographs and analyses patterns within the radiographs taken [11]. 2019 also saw the introduction of Kapanu by Ivoclar Vivadent which uses propriety software used to visualize the possible smile outcome of the patient's post-treatment [11].

Now to further discuss how Artificial Intelligence does practically function or how the computer does identify with human cognitive skills, here is the relationship between Algorithmic patterns and Artificial intelligence. [15].

Algorithms and artificial intelligence

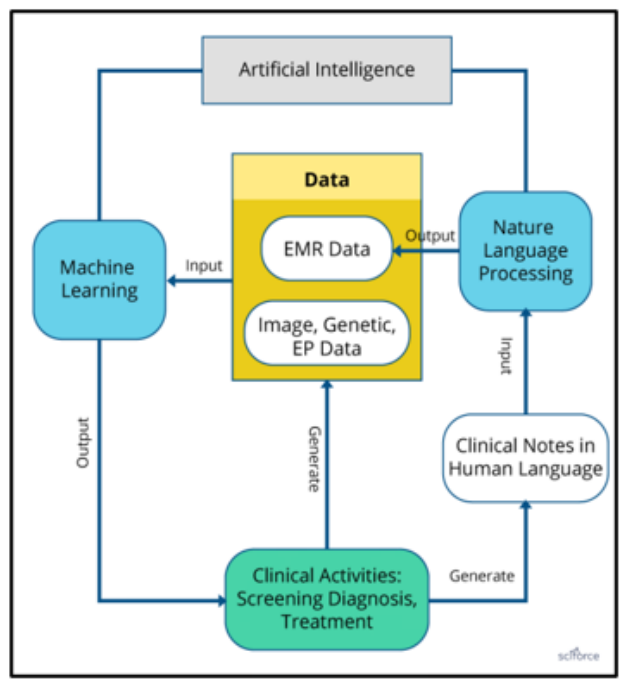

Figure 1: Machine learning and natural processing in healthcare.

Algorithms form the backbone for Artificial intelligence. Despite the variety of applications of Artificial intelligence, in the clinical studies and healthcare services, they fall into two major categories:

- Analysis of structured data: This includes data related information about genes; biomarkers and other associated images.
- Analysis of unstructured data: This includes notes; medical journals or patient's surveys to complement the structured data.

Now the approach to both the data system is quite different [15]

While the idea of analysis for structured data is fueled by Machine learning and Deep learning algorithms that of unstructured data is fueled by Specialized Natural Language Processing $[12,13,15]$.

\section{Machine learning algorithms [12]}

These extract features from data such as patient traits and medical outcomes of interest. Now in literature, there are several algorithms supporting this idea however the most common ones are Support Vector Learning [SVM] and Neural networking.

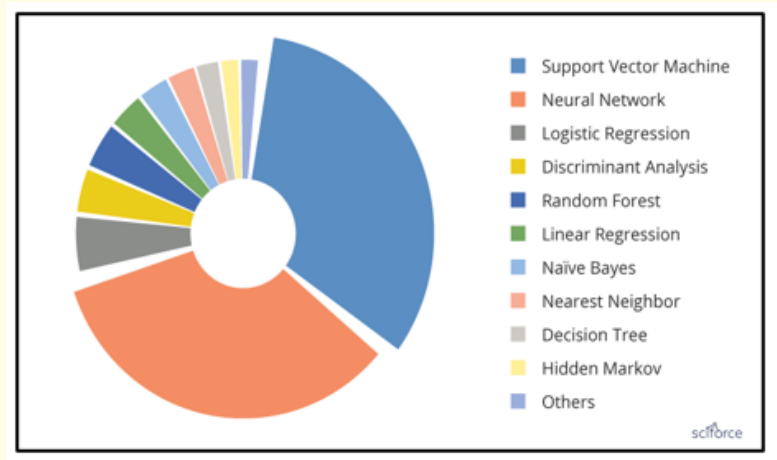

Figure 2: The most popular machine learning algorithms used in medical literature.

The idea of Support Vector Learning [SVM] [12] puts forth a concept in which algorithms are used for classification or regression of a given data set based on a given common hyper plane. To put in simple words the computer tries to classify and divide the data set on basis of already present and given characteristics. Whereas Neural Networks [13-15] process via an association of input and output variables through the help of hidden layers of pre-specified functionals. Now it is this hidden layer that forms an integral part of data processing.

Example: To simply put if I (user) make a machine to classify tomatoes and cherries, in the case of Support vector theory the user 
needs to intervene and characterize the specifics of tomatoes and cherries. Thereby creating an algorithm that would help to separate the data on basis of the characteristics the user has mentioned (now this idea is extremely useful in cases of clinical research and diagnostic processing).

Whereas in the case of a Neural network the user does not need to specifically intervene, the computer processes the data based on the information fed and prior training done, the computer instantly recognizes the object through various attributes such as the pixels (28 x 28 dimensional property) being fed into the input layer that then passes through multiple hidden layers (with bias and functions) via channels and finally giving the result in form of output.

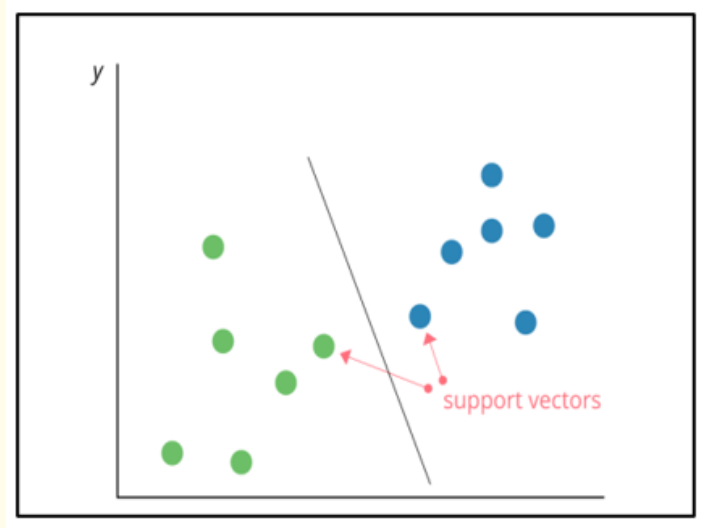

Figure 3: Support vector machine.

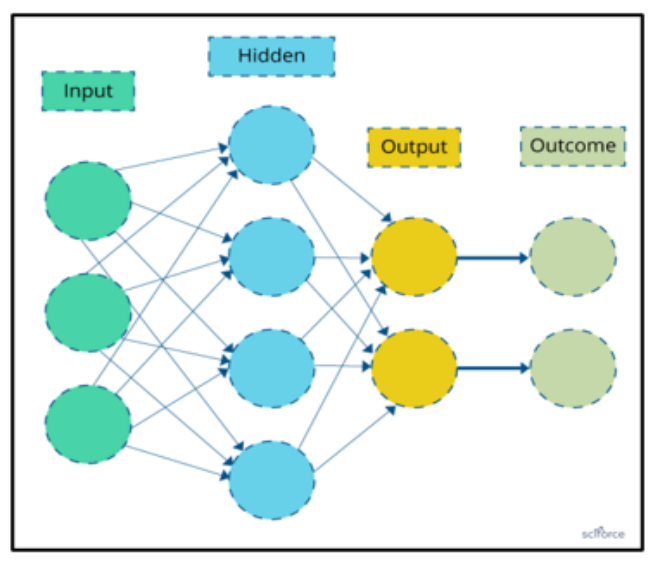

Figure 4: Neural networks.

\section{Deep learning algorithms}

Is an example of a classical neural networking technique using more and extended hidden layers, therefore, having an increased capacity than the classical machine learning techniques. It can navigate through deep and complex nonlinear patterns of data. In medical and healthcare uses deep learning algorithms to combine the benefits of both machine learning and natural language processing tasks. The most common ones used in medicine are [1315]:

1. Convolutional neural network

2. Recurrent neural network

3. Deep belief network

4. Multilayer perceptron.

\section{Natural language processing}

Now a piece of huge information around $80 \%$ of the data presents in an unstructured form this includes laboratory tests; narrative texts; operative notes and discharge summaries. These unstructured data are incomprehensible to the computer and lag as a result of the human workload. This is where Natural Data Processing comes into the picture.

Example: If I go to a salon and ask the receptionist about my appointment, the lady says "The hairstylist is free!" now although I (user) understand the context and thereby can conclude that "The hairstylist is free for my appointment". However, the computer does not on its own understand the given context and thereby can conclude several unfavorable possibilities. Here is where Natural language processing steps in, it analyses the data and helps to form a favorable context for the given purpose thereby supporting decision making.

This is how virtual support systems such as SIRI and ALEXA work; through understanding the context of the user and his/her requirements thereby performing the required task by the user.

\section{Cognitive computing}

At the given moment hidden among the 2.5 billion gigabytes of data being processed each day are increasingly complex patterns that are incomprehensible to interpret. We need systems that can interact with us more intelligently and help us understand the data in form of feedbacks. In general term, Cognitive Computing is referred to any hardware or software which mimics the human brain and helps to improve human decision making. 
Example: Take a doctor who doesn't have time to continuously absorb a vast, ever-changing body of medical research, with the use of Cognitive Assistant they can question and interact within seconds and streamline everyday tasks and get more accurate processed knowledge of the research materials required for the task.

\section{Artificial intelligence versus oral medicine}

The idea of capturing human minds and mimicking their skills has always been an enigma to scientists and philosophers. Although Artificial Intelligence has made its due progress since its inception, we have merely just scratched the surface. On the one hand, while it's currently shaping our existing relationship with medical science while on the other it's also considered an eminent threat in many fundamental ways for the near future.

How is artificial intelligence shaping oral medicine?

Every year an estimated 3.58 billion individuals complain of Dental Caries averaging around 5\% of the total health expenditure in most of the countries [16]. At present algorithms such as those of Dentistry.AI are at its final stages of testing and shall be deployed in a few years as commercially trained caries detecting agents powered completely by Artificial Intelligence [16]. One should not forget that in India alone around 20 individuals per 1 lakh population are affected by oral cancer; in these cases adequately trained neural networks can be a boon to a clinician to confirm the early diagnosis and predict favorable prognosis [16]. Moreover, analysis of data and individual profiles can predict a possible genetic predisposition for oral cancers. Envision having an Artificial Intelligence assistant that has learned the art of recognizing errors and significant diagnostic points via learning from millions of 3D images and $\mathrm{x}$-rays. Imagine how early screening and classifying of altered oral mucosa for premalignant and malignant conditions would result in early diagnosis and better prognosis of oral pathologies. We have all as dentists studied about how our oral cavity is a mirror to numerous systemic problems, Artificial Intelligence can further approximate gaps to clarify the interrelationship of general disease pattern and their mutual influence on oral health. It can learn from a large volume of healthcare data and train itself to be a competent risk assessing agent. It practically reduces observational error and at the same time reduces the risk of observational fatigue. In all ways, it opens up doors for a vast plethora of choices ranging from developing precision medicines and formulating new drug patterns to analyzing medical records and assisting physicians with their cognitive learning capabilities.
How is artificial intelligence a threat?

As Artificial intelligence is pushing us towards a digital revolution there is an argument stating that how it will replace human jobs [17]. It also raises serious questions on ethics and responsibilities. Complex algorithms mean it requires more training, right from healthcare students and professors to individuals handling the day to day operations all need to be trained with changes in existing curriculums to adapt to the digital revolution. One must also understand that these complex programming skills require profound knowledge and specialization in given computational arenas which becomes difficult to come across. The entire process at the same time increases the amount of investment. While we may all harp about the idea of digitalization in this field, in reality, most of the products are not available for commercial use and only a few have made inroads into commercial markets.

Artificial Intelligence in healthcare and growing market trends

According to Accenture analytics by the year 2021 Artificial Intelligence-powered healthcare could generate 6.6 billion dollars of revenue and up to 150 billion dollars of revenue by the year 2026 for counties like the US [18-23].

The report mentioned the following breakup of revenue.

\begin{tabular}{|l|l|}
\hline \multicolumn{1}{|c|}{ Application } & \multicolumn{1}{c|}{ Value } \\
\hline Robot-Assisted Surgery & $\$ 40 \mathrm{~B}$ \\
\hline Virtual Nursing Assistant & $\$ 20 \mathrm{~B}$ \\
\hline Administrative Workflow Assistance & $\$ 18 \mathrm{~B}$ \\
\hline Fraud Detection & $\$ 17 \mathrm{~B}$ \\
\hline Dosage Error Reduction & $\$ 16 \mathrm{~B}$ \\
\hline Connected Machines & $\$ 14 \mathrm{~B}$ \\
\hline Clinical Trial Participant Identifier & $\$ 13 \mathrm{~B}$ \\
\hline Preliminary Diagnosis & $\$ 5 \mathrm{~B}$ \\
\hline Automated Image Diagnosis & $\$ 3 \mathrm{~B}$ \\
\hline Cyber security & $\$ 2 \mathrm{~B}$ \\
\hline Total & $\sim \$ 150 \mathrm{~B}$ \\
\hline
\end{tabular}

Table: Source: Accenture analysis "Value" is the estimated potential annual benefits for each application by 2026 .

The given data [23] clearly shows how the adoption of Artificial Intelligence-powered healthcare science represents an opportunity for industry players to capitalize on this new ever-changing 
digital landscape. The report correctly mentioned the idea of how "Artificial Intelligence thinks for itself and pays for itself too" [23]. At the same time, this technology is also addressing the inequality of clinician demand and supply which has been widening over the years [21].

\section{Robotics and dentistry}

On March 2 ${ }^{\text {nd }}, 2017$ Neocis in Florida announced that the company had received U.S Food and Drug Administration clearance to market Yomi [29]. This was path-breaking for its time as Yomi became the first surgical robot to be used in Dentistry that could assist in both planning (pre-operative phases) and surgical (intraoperative) [24] phases of dental implantation surgery via providing navigational guidance for the surgical instruments. Ultra-realistic robotic models such as Showa Hanako and Simroid [25,26] are currently being used to generate typical human-like gestures and responses allowing dental students to experience working with patients. Another robotic family the Geminoid family [25,26] has introduced Geminoid- F robotic series which presents with better motion capture facilities and replicates human facial expressions. Others such as HRP-4 variants can also sing and mimic human sounds. Also under developments are Endo micro robots which have been introduced recently to reduce the problems under conventional endodontic therapies. On the other side is the Dental Nanorobots [27] which have a huge scope of application starting from the usage of nanotechnology: to administer local anesthesia, as a permanent cure for hypersensitivity, to support for complete orthodontic realignment in a single visit, allowing covalently bonded diamond zed enamel and assisting in continuous oral health maintenance using Mechanical Dentifrobots [25]. Dental Nanorobots could be used to destroy caries causing bacteria or to repair tooth blemishes where decay has set in, using the computer to direct these tiny workers in their task.

Artificial intelligence and its contribution to healthcare in the current pandemic

The current pandemic is the worst public health disaster of the $21^{\text {st }}$ century, at present numerous Artificial Intelligence-driven projects depending on data science and health sciences are helping to envisage; clarify; handle and support critical decision makings. As the pandemic progresses Artificial Intelligence-powered algorithms are required to forecast the outburst of the causative agent through data reports thereby contributing to ineffective govern- ment policies. At the same time, the modern computing architecture allows the database to be available to different institutes for allowing them to develop autonomous or collaborative models to handle various tasks thereby training Natural Language data processing units to flag their priorities [30-32]:

- $\quad$ Artificial Intelligence-driven algorithm BlueDot not only confirmed and predicted the outbreak of the Zika Virus in Florida but also predicted the pattern of the current COVID pandemic 19 days before the announcement of WHO.

- Researchers from Huazhong University and Tongji Hospital in Wuhan have developed XGBoost machine -learning based prognostic model that can quickly analyze blood samples to predict infection rate with $90 \%$ success rate.

- COVID-Net a deep learning model is designed to detect COVID 19 positive cases from chest x-rays.

- License, a contactless syndromic surveillance platform is used to forecast seasonal flu and other viral outbreaks.

- Artificial Intelligence-powered humanoid robots CloudGinger (aka XR-1) are used to assist healthcare workers in the healthcare facilities.

- In India C-Astra; Mitra and RoboDoc by Invento Robotics are currently being used to assist in public places to help guide social distancing policies.

- Asimov Robotics; a Kochi-based startup has deployed two robots at the beginning of March this year to help spread awareness about coronavirus.

- CO-BOT and NIGA -BOT are two low-cost robots developed by ITI Cuttack are been currently deployed at healthcare facilities. Moreover, an indigenous Delhi-based robot Prithvi is also currently been used for the same.

- In April Milagrow iMap 9 and Humanoid ELF were deployed by AIIMS for its COVID-19 wards.

\section{Conclusion}

Ever since the beginning of time, the human mind has been a center of discussion in numerous theories and ideas, and as we shape our way for the future a fundamental question arises; "Is it possible to train our computers and build super realistic machines which could wipe out troubles faced by mankind today?" This very statement provides the scope of artificial intelligence. However, in 
the Dartmouth Workshop of 1956, it was mentioned "Every aspect of learning or any other feature of intelligence can be so precisely described that a machine can be made to simulate it". This very statement puts the future of artificial intelligence in question as there are practical limits to the abilities of computers and there are specifics of human minds that cannot be replicated. As healthcare keeps on growing every day and new challenges such as the current pandemic jeopardize our growth we need these machines with intellect to help be our frontline agents. However one must remember that healthcare is a multidisciplinary approach and thinking about replacing its task load with mere algorithms seems more of fiction than reality.

\section{Bibliography}

1. Modern English textbooks and translations prefer "theory of Form" to "theory of Ideas", but the latter has a long and respect tradition starting with Cicero and continuing in German philosophy until present, and some English philosophers prefer this in English too. See W. D. Ross, Plato's Theory of Ideas (1951).

2. The edge of reason: understanding neural networks through Plato's Theory of forms.

3. The name of this aspect of Plato's thought is not modern and has not been extracted from certain dialogues by modern scholars. However, it is attributed to Plato without any direct textual evidence that Plato himself holds the views of the speakers of the dialogues. The term was used at least as early as Diogenes

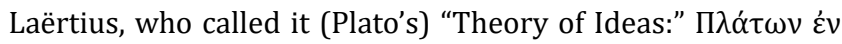

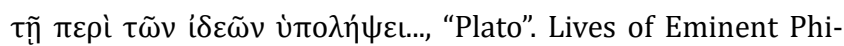
losophers. Book III. p. Paragraph 15.

4. Solomonoff RJ. "The Time Scale of Artificial Intelligence; Reflections on Social Effects, Human Systems Management 5 (1985): 149-153.

5. Kline Ronald R. "Cybernetics, Automata Studies and the Dartmouth Conference on Artificial Intelligence, IEEE Annals of the History of Computing, October-December 2011, IEEE Computer Societ (2011).

6. (Turing 1950, p. 442) Turing does not call his idea "Turing test", but rather the "Imitation Game"; however, later literature has reserved the term "Imitation game" to describe a particular version of the test. See \#Versions of the Turing test, below. Tur- ing gives a more precise version of the question later in the paper: "[T] hese questions [are] equivalent to this, 'Let us fix our attention on one particular digital computer $C$. Is it true that by modifying this computer to have an adequate storage, suitably increasing its speed of action, and providing it with an appropriate programme, $\mathrm{C}$ can be made to play satisfactorily the part of $A$ in the imitation game, the part of $B$ being taken by a man?'” (Turing (1950): 442

7. “Alan Turing at 100". Harvard Gazette. Retrieved 2016-02-22.

8. Berk AA. "LISP: the Language of Artificial Intelligence. New York: Van Nostrand Reinhold Company, 1985. 1-25. Lederberg, Joshua. An Instrumentation Crisis in Biology. Stanford University Medical School. Palo Alto (1963).

9. "Robotic Surgery" (2011).

10. “Robot-Assisted Surgery: da Vinci” (2011).

11. "How is artificial intelligence shaping dentistry" Lou Shuman, DMD, CAGS (2019).

12. "Machine Learning textbook" (2020).

13. "Deep Learning".

14. Chen Yung-Yao., et al. "“Design and Implementation of Cloud Analytics-Assisted Smart Power Meters Considering Advanced Artificial Intelligence as Edge Analytics in Demand-Side Management for Smart Homes". Sensors 19.9 (2019): 2047.

15. Top AI algorithms for healthcare (2019).

16. "Study on patient's awareness towards Role of Artificial Intelligence In Dentistry" Dr.Manisha Jiaswal ; Dr. Neena Gupta and Akansha Singh original research article published in the IJHSR.

17. Susskind R and Susskind D. "Technology will replace many doctors, lawyers and other professionals. Harvard Business Review cited oct 16 (2016).

18. Accenture; "Why Artificial Intelligence Is the Future of Growth,".

19. Frost and Sullivan.

20. CB Insights; "From Virtual Nurses To Drug Discovery: 106 Artificial Intelligence Startups In Healthcare;” posted (2017). 
21. Accenture. "Digital Health Tech Vision 2016: Liquid Workforce (2016).

22. "Accenture: Artificial Intelligence Healthcare Market to Reach $\$ 6.6 B$ by 2021" by Fred Pennic (2021).

23. Artificial Intelligence: Healthcare’s New Nervous System.

24. The First Surgical Robot Designed For Dental Implant, Yomi Surpasses 1000 Implants (2020): 00.

25. Rawtiya M., et al. "Application of robotics in dentistry". Indian Journal of Dental Sciences 6 (2014): 1700-1706.

26. Ishiguro H and Geminoid DK. "Geminoid series (2010).

27. Shetty NJ., et al. "Nanorobots: Future in dentistry". Saudi Dental Journal 25 (2013): 49-52.

28. Bansal A., et al. "Robots in head and neck surgery". Journal of Applied Dental and Medical Sciences 2 (2016): 168-175.

29. Yomi, theFirst Robotic Dental Surgery System Now Cleared by FDA.

30. "How artificial intelligence is transforming the world in the current pandemic" By Ethan Miller (2020).

31. "Artificial Intelligence during a pandemic: The COVID-19 example” by: Santhian Dananjayan; Gerard Marshall Raj ON 20TH MAY (2020).

32. "These Made-in-India robots are helping health workers fight COVID-19" By Trisha Medhi (2020).

\section{Assets from publication with us}

- Prompt Acknowledgement after receiving the article

- Thorough Double blinded peer review

- Rapid Publication

- Issue of Publication Certificate

- High visibility of your Published work

Website: www.actascientific.com/

Submit Article: www.actascientific.com/submission.php

Email us: editor@actascientific.com

Contact us: +919182824667 